\title{
Advances in design and application of neural networks
}

\author{
Guest editors: Robert Howlett, Ignac Lovrek, Lakhmi Jain, Chee-Peng Lim, \\ and Bogdan Gabrys
}

\author{
Lakhmi C. Jain
}

Published online: 12 February 2010

(C) Springer-Verlag London Limited 2010

Even since the introduction of backpropagation in 1986, neural networks have gained considerable attention from researchers for more than two decades now. A variety of neural network models have been designed and applied successfully to solve real-world problems in various domains. Neural networks have also been widely synergized with other machine learning and complementary techniques to achieve improvements in robustness, adaptivity, and applicability. In this special issue, a total of seven articles, based on extended papers from the 12th International Conference on Knowledge-Based and Intelligent Information \& Engineering Systems (KES2008) as well as from other submissions, are presented. These papers form a small sample of research in demonstrating the usefulness of intelligent information processing techniques in the design and application of neural networks. A summary of each paper is as follows.

In the first article, an approach based on the decomposition of the square approximation algorithm for updating neural network weights is proposed. The proposed approach is an improvement of gradient methods and has a fast convergence rate. The approach is inherently parallel, hence enabling its implementation on the computer grid. To evaluate the effectiveness of the proposed approach, a series of experiments using the Multi-Layer Perceptron neural network with varying parameters is conducted. The experimental results indicate time savings in multiple thread execution for a wide range of network parameters.

L. C. Jain $(\square)$

School of Electrical and Information Engineering, University of South Australia, Mawson Lakes Campus, Adelaide, SA 5095, Australia

e-mail: Lakhmi.Jain@unisa.edu.au
A Design of Experiments approach, i.e., the Taguchi technique, to determine the optimal settings of neural network models for process monitoring and fault detection and isolation tasks is described in the second article. The auto-associative artificial neural network coupled with nonlinear principal component analysis is employed for experimentation. The important network parameters are captured in a joint orthogonal array (OA) such that the interactions between noise and control process variables can be considered. The results show the usefulness of the Taguchi method in realizing a reliable scheme for tackling fault detection and isolation problems.

Methods to extend and enhance the Self-Organizing Background Subtraction (SOBS) algorithm are proposed in the third article. The background model constructed and maintained in SOBS is based on a self-organizing neural network organized as a two-dimensional grid of neurons. To further enhance the SOBS algorithm, a fuzzy rulebased approach is introduced. The aim is to introduce, during the update phase of the background model, an automatic and data-dependent mechanism for further reinforcing into the background model the contribution of pixels that belong to. It is shown that the proposed fuzzy approach improves the accuracy of the corresponding crisp moving object detection procedure and performs better than other methods.

In the fourth article, a multivariable adaptive controller based on a dynamical neural network used as a model of the unknown plant is developed. An input-output feedback linearization technique is realized in the controller design. The Lyapunov approach is used to formulate the adaptation laws of the neural network weights. To demonstrate the applicability of the proposed neural controller, a simulation study on a complex nonlinear and time-varying wastewater depollution bioprocess is conducted. The results indicate 
that the proposed controller is useful for practical situations whereby the process nonlinearities are not completely known, and the process dynamics vary with time.

The fundamental principles of the functional link neural network are explained in the fifth article. A road map on functional link neural network models is presented. In addition, a new hybrid learning scheme based on particle swarm optimization and back-propagation for the functional link neural network with the orthogonal Chebyshev polynomials is proposed. Applicability of the proposed network to data classification tasks is evaluated. Benchmark data sets are used, and the results are compared with those from other approaches. The results demonstrate the advantages of the proposed network in undertaking data classification problems.

A hybrid neural network based on the fuzzy ARTMAP model and the quality threshold clustering principle is introduced in the sixth paper. The aim of the hybrid network is twofold, i.e., to minimize the effects of sequence of training data presentation in fuzzy ARTMAP and to improve its classification performance. A series of experiments using benchmark medical data sets is used to evaluate the effectiveness of the proposed model. The results show that the proposed network is able to produce good classification performances when compared with those from other methods. Moreover, the performance of the proposed network is not affected by the training data sequences.

In the seventh article, the issue of visual landmark recognition in autonomous robot navigation is addressed. The Adaptive Resonance Theory (ART)-based neural network is developed real-time recognition of visual landmarks in unmanned ground vehicles. A series of experiments using real images is conducted, and the results show that the proposed model is able to recognize objects as well as partially occluded landmarks in clean and cluttered backgrounds. The real-time landmark recognition capability of the proposed model is demonstrated using various autonomous robot navigation trials, and positive results have been achieved in both indoor and outdoor environments.

The guest editors are very grateful to the authors for their contributions and to the reviewers for their time and effort in reviewing the manuscripts, as well as to the editorial team of Neural Computing and Applications for their support and help. 